\title{
Irregular eating of meals in adolescence and the metabolic syndrome in adulthood: results from a 27 -year prospective cohort
}

\author{
Maria Wennberg ${ }^{1, *}$, Per E Gustafsson ${ }^{1}$, Patrik Wennberg ${ }^{2}$ and Anne Hammarström ${ }^{1}$ \\ ${ }^{1}$ Social Medicine, Public Health and Clinical Medicine, Umeå University, 90187 Umeå, Sweden: ${ }^{2}$ Family Medicine, \\ Public Health and Clinical Medicine, Umeå University, Umeå, Sweden
}

Submitted 3 December 2014: Final revision received 11 March 2015: Accepted 1 April 2015: First published online 4 May 2015

\begin{abstract}
Objective: The objective was to investigate whether irregular eating of meals in adolescence predicts the metabolic syndrome and its components in adulthood, and if any specific meal is of particular importance.

Design: Prospective cohort study with 27 years of follow-up. Information on meals (breakfast, school lunch and dinner with family), lifestyle (alcohol consumption, smoking habits, physical activity, consumption of sweets and pastries) at age 16 years was assessed from questionnaires, and presence or not of the metabolic syndrome and its components were defined at age 43 years in 889 participants $(82 \cdot 1 \%$ of total cohort). Logistic regression was used to calculate odds ratios and confidence intervals.

Setting: The Northern Swedish Cohort; all school-leavers of the 9th grade in the town Luleå in 1981.

Subjects: Adolescents (age 16 years).

Results: Irregular eating of meals at age 16 years was associated with higher prevalence of the metabolic syndrome at age 43 years (OR $=1 \cdot 74 ; 95 \%$ CI $1 \cdot 12$, $2 \cdot 71$ ), but this was explained by concurrent unhealthy lifestyle at age 16 years. Poor breakfast at age 16 years was the only meal associated with the metabolic syndrome at age 43 years, independent of other meals, BMI $\left(\mathrm{kg} / \mathrm{m}^{2}\right)$ and lifestyle at age 16 years $(\mathrm{OR}=1 \cdot 67 ; 95 \%$ CI 1.00, 2.80).

Conclusions: Irregular eating of meals in adolescence predicted the metabolic syndrome in adulthood, but not independently of BMI and lifestyle in adolescence. Poor breakfast in adolescence was the only specific meal associated with future metabolic syndrome, even after adjustments. Breakfast eating should be encouraged in adolescence.
\end{abstract}

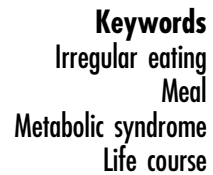

Keywords Meal Life course
A regular meal pattern with breakfast, lunch and dinner as main meals and one to three snacks in between is often recommended as part of a healthy lifestyle ${ }^{(1)}$, but the longterm health effects of deviating from regular eating patterns are largely unknown. Skipping meals is common and by some people considered a simple way to lower energy intake ${ }^{(2)}$.

A number of cross-sectional studies suggest that eating irregularly may involve increased risk for cardiometabolic disease. For example, cardiometabolic risk factors have been found to be less prevalent in regular eaters (those regularly eating breakfast, lunch and dinner or having a higher meal frequency) compared with irregular eaters, both in young and older adults ${ }^{(3-5)}$. More frequent daily eating occasions have been associated with a higher diet quality ${ }^{(4)}$ and better appetite control ${ }^{(6)}$. Randomized crossover trials suggest that irregular meal frequency may cause insulin resistance ${ }^{(7)}$.
Previous cross-sectional studies have found associations between irregular eating and prevalence of the metabolic syndrome, a cluster of risk factors associated with cardiovascular risk and early death, in adulthood ${ }^{(3,8)}$.

So, while there is evidence of short-term health risks from irregular eating, there is a lack of prospective studies investigating the long-term effect of irregular eating. Here, a life-course perspective is helpful to take into account that exposure during early life may involve enduring health risks into adulthood, e.g. the possible effect of regularity in meals in adolescence on cardiometabolic risks in adulthood. We have recently shown that lifestyle factors at age 16 years, such as sedentary behaviour ${ }^{(9)}$ and poor breakfast habits ${ }^{(10)}$, predict the metabolic syndrome at age 43 years.

The aim of the present study was to investigate if overall irregular eating of meals (a summary of breakfast, school 
lunch and dinner with family) in adolescence (age 16 years) predicts prevalence of the metabolic syndrome in adulthood (at age 43 years), and if any specific meal habit is of particular importance.

\section{Methods}

\section{Population and setting}

The Northern Swedish Cohort consists of all school-leavers of the 9 th (final) grade (age 16 years) in the municipality of Luleå in $1981(n \text { 1083 })^{(11)}$. Participants filled out an extensive questionnaire concerning lifestyle, socioeconomic condition and health. Follow-ups were conducted in 1983 (age 18 years), 1986 (age 21 years), 1995 (age 30 years) and 2008 (age 43 years), resulting in a follow-up time of 27 years. Health examinations were performed at ages 16, 21 and 43 years. The health examination at 43 years was performed by medical personnel at the participants' respective health-care centre, making classification of the metabolic syndrome possible. The participation rate has been extremely high; in 2008, $94 \%$ of the baseline individuals still alive participated ${ }^{(12)}$. A sample of 889 participants was made for the present study, for which we have data on metabolic syndrome and meal habits $(82 \cdot 1 \%$ of total cohort).

The cohort has been found to be representative of similar age cohorts in Sweden as a whole in relation to demographics, health conditions and socio-economic variables $^{(11)}$.

\section{Measurements}

Breakfast at age 16 years

Breakfast habits were inquired in the questionnaire at age 16 years: 'What did you have for breakfast this morning?' Participants were instructed to underline what they had eaten the same morning, from a list with food items divided into seven different food groups normally eaten for breakfast (drinks, milk products, egg/meat/fish, fruit/ vegetables, porridge/cereals, dark bread, white/sweet bread). The alternative 'something else' accounted for food items that could have been missed. Those reporting not eating anything at all for breakfast were defined as 'breakfast skippers' ( $n$ 66), and those reporting only drinking something energy-containing ( $n$ 12) or only eating something sweet ( $n$ 10), such as a bun, cookie or biscuit, were treated as a separate category ('drink or sweet for breakfast'; $n$ 22). Due to low frequencies in these sub-categories and similar point estimates in the association with the metabolic syndrome, these categories, both representing poor breakfast habits, were collapsed into a larger 'poor breakfast habits' category in the main analysis ( $n$ 88). This group is defined as 'not eating breakfast' and coded as 0 , whereas those defined as eating breakfast were coded as 1 .
School lunch at age 16 years

In Sweden, a cooked, nutritious meal, free of charge, is offered for lunch in the school restaurant to all children attending compulsory school. There was one question on frequency of eating school lunch in the questionnaire at 16 years, with the answer alternatives (i) 'every school day', (ii) 'skips school lunch one day a week', (iii) 'skips school lunch several days a week' and (iv) 'seldom eat school lunch'. Those reporting to eat school lunch every school day were coded as 1 and the other answer alternatives resulted in a code of 0 .

\section{Dinner with family at age 16 years}

There was one question concerning frequency of eating dinner with family in the questionnaire at 16 years, with the answer alternatives (i) 'I do not eat dinner', (ii) 'every or almost every day', (iii) 'several days a week', (iv) 'one day every week' and (v) 'more seldom'. Those reporting having dinner with their family every or almost every day were coded as 1 and the other answer alternatives resulted in a code of 0 .

\section{Meal regularity at age 16 years}

Regularity in the various meal habits of breakfast, school lunch and dinner with family at age 16 years was combined into a three-category variable: (i) all meals regularly ( $n$ 439); (ii) one meal irregularly ( $n$ 332); and (iii) two or all meals irregularly $(n$ 115). Those with two or all three meals irregularly were combined due to few individuals in the group with all three meals irregularly ( $n 15)$.

Metabolic syndrome and its components at age 43 years Waist circumference was measured in indoor clothing to the nearest $0.5 \mathrm{~cm}$. Blood samples were assessed for TAG, HDLcholesterol and glucose according to the laboratory routines at the Department of Clinical Chemistry, Umeå University. $\mathrm{CV}$ at high/low concentration was $1.5 / 1.2 \%$ for glucose, $1.7 /$ $1.5 \%$ for TAG and $2 \cdot 8 / 2 \cdot 8 \%$ for HDL-cholesterol. External quality control was managed by EQUALIS (External Quality Assurance in Laboratory medicine In Sweden). Blood pressure was measured with a standard sphygmomanometer after resting for at least $5 \mathrm{~min}$, on the right arm, in sitting position. The mean of two measures was used.

Presence of the metabolic syndrome and its components was operationalized as in our previous reports ${ }^{(12,13)}$. According to the International Diabetes Federation definition $^{(14)}$, for a person to be defined as having the metabolic syndrome they must have central obesity (waist circumference $\geq 80 \mathrm{~cm}$ for women or $\geq 94 \mathrm{~cm}$ for men); plus two or more of the following four components: (i) increased TAG $(\geq 1.7 \mathrm{mmol} / \mathrm{l})$ or treatment for that lipid abnormality; (ii) reduced HDL-cholesterol $(<1.29 \mathrm{mmol} / \mathrm{l}$ for women or $<1.03 \mathrm{mmol} / \mathrm{l}$ for men) or treatment for that lipid abnormality; (iii) increased blood pressure (systolic blood pressure $\geq 130 \mathrm{mmHg}$ or diastolic blood pressure $\geq 85 \mathrm{mmHg}$ ) or treatment of hypertension; and 
(iv) increased fasting glucose $(\geq 5.6 \mathrm{mmol} / \mathrm{l})$ or diagnosed type 2 diabetes. Information on medication was selfreported. Current type 2 diabetes was based on selfreported diabetes at age 43 years, excluding those who also reported diabetes at age 30 years, who were regarded as having type 1 diabetes.

Both the metabolic syndrome and its components were used as binary outcomes ( $1=$ presence, $0=$ absence) in the analyses.

\section{Assessment of confounders at age 16 years}

Daily consumption of pure alcohol was estimated from a validated battery of questions (frequency and amount of medium-strength beer, strong beer, wine and spirits). Participants above the sex-specific 80th percentile of alcohol consumption were classified as high consumers. Smoking habits were classified as current smoker or not (including ex-smokers). A reliability test-retest of the alcohol and tobacco questions was conducted at age 16 years, within a 2-month period, showing that similar answers were given by the same pupils to the same questions ${ }^{(15-17)}$. Those who reported participation in physical activity more often than once per week were classified as physically active. Daily consumption of sweets and pastries was defined as high consumption of these food groups. Family history of diabetes was defined as a parent or sibling with diabetes, and the information was assessed from the questionnaire at age 16 years. Social class in adolescence was operationalized as in previous reports ${ }^{(12,18)}$. Social class was defined based on parents' occupation when the participant was aged 16 years (both parents as manual workers counted as low social class). Height and weight from school health records were used to calculate BMI $\left(\mathrm{kg} / \mathrm{m}^{2}\right)$.

\section{Statistical analyses}

To examine potential selection bias, those excluded because of incomplete measures of the metabolic syndrome ( $n$ 107), any missing data for meal regularity ( $n$ 8) or missing data on any of the covariates at age 16 years ( $n$ 20) were compared for exposures (regularity in breakfast, school lunch and dinner with family) and covariates (sex, BMI, alcohol, smoking status, physical activity, consumption of sweets and pastries, parents' socio-economic status and family history of diabetes) at age 16 years with those included in the study. The $\chi^{2}$ test was used for categorical variables and the Kruskal-Wallis non-parametric $t$ test for continuous variables. In the excluded group there was a higher proportion not eating school lunch regularly compared with the included group (54\% v. $41 \%, P=0.010)$, as well as a higher proportion not eating dinner with family regularly (21\% v. $14 \%$, $P=0 \cdot 045)$. There were no other statistically significant differences in proportion of meal habits or covariates between those excluded and those included in the study.

Baseline characteristics were compared between those classified as eating (i) all meals regularly, (ii) one meal irregularly or (iii) two or all meals irregularly, using the $\chi^{2}$ test or Kruskal-Wallis non-parametric test.

Spearman's rank correlations $\left(R_{\mathrm{S}}\right)$ were calculated between regularity in the specific meals and covariates.

To examine the impact of overall meal regularity at age 16 years on risk of the metabolic syndrome at age 43 years, binary logistic regression was performed with those reporting eating all meals regularly as reference category. The following covariates at age 16 years were added in the multivariable models: in model 1, sex (because the prevalence of the metabolic syndrome differs between men and women; the prevalence is higher in men in this population (34\% in men $v .19 \%$ in women) as well as in other European populations ${ }^{(19)}$, but not consistently so worldwide ${ }^{(20)}$ ); in model 2 , model 1 plus BMI (pre-existing metabolic situation associated with meal regularity at age 16 years); and in model 3, model 2 plus high alcohol intake, smoking status, physical activity, daily consumption of sweets and pastries (lifestyle covariates associated with meal regularity at age 16 years). An additional model adding social class and family history of diabetes to model 3 was built to rule out pre-existing metabolic or social confounding. $P$ for trend was calculated for having none, one, two or three irregular meal habits. To examine the impact of specific meal regularity (breakfast, school lunch or dinner with family) at age 16 years on risk of the metabolic syndrome at age 43 years, binary logistic regression was performed with those reporting eating the specific meal regularly as reference category. We added the other two meals in model 1, sex in model 2, BMI in model 3 and high alcohol intake, smoking status, physical activity and daily consumption of sweets and pastries in model 4. An additional model was built adding social class and family history of diabetes to model 4. Sensitivity analyses were performed in which dichotomized covariates were replaced with continuous ones if possible (alcohol consumption in quintiles, exercise as times/week, consumption of sweets and pastries as times/week).

Crude and adjusted odds ratios for the components of the metabolic syndrome at age 43 years by overall meal regularity were calculated with those reporting eating all meals regularly as reference category, using the same multivariable models as for the metabolic syndrome. Also using the same multivariable models, crude and adjusted odds ratios for the components were calculated for irregularity in the specific meals, with those classified with regular consumption of the meal as reference category.

The statistical computations were carried out using the statistical software package IBM SPSS Statistics for Windows version 22 .

\section{Results}

Those reporting eating all meals regularly at age 16 years reported concurrent healthier lifestyle; a lower proportion 
were smokers, had high alcohol intake and daily intake of sweets and pastries, whereas a higher proportion were physically active more than once per week. Higher BMI was associated with eating fewer meals regularly (Table 1).

Regularity in all specific meal habits correlated significantly with one another; for school lunch and breakfast Spearman's rank correlation $\left(R_{\mathrm{S}}\right)$ was $0.18, P<0.001$, but dinner with family correlated only weakly with breakfast and school lunch $\left(R_{\mathrm{S}}=0.09, P=0.005\right)$. All specific meal habits correlated negatively with concurrent smoking and alcohol consumption. Breakfast and school lunch correlated positively with concurrent physical activity and school lunch correlated negatively with concurrent consumption of sweets and pastries (Table 2).

Analyses on the relationship between meal regularity in adolescence and the metabolic syndrome in adulthood are shown in Table 3. Eating two or all meals irregularly at age 16 years was associated with higher prevalence of the metabolic syndrome at age 43 years in sex-adjusted analysis (model 1), compared with eating all meals regularly. This association was attenuated and no longer statistically

Table 1 Baseline characteristics for participants from the Northern Swedish Cohort, according to meal regularity (breakfast, school lunch, dinner with family) at age 16 years

\begin{tabular}{|c|c|c|c|c|c|c|c|c|c|c|}
\hline & \multicolumn{3}{|c|}{ All meals regularly } & \multicolumn{3}{|c|}{ One meal irregularly } & \multicolumn{3}{|c|}{ Two or all meals irregularly } & \multirow[b]{2}{*}{$P$ for trend } \\
\hline & $n$ & $\%$ or median & P25, P75 & $n$ & $\%$ or median & P25, P75 & $n$ & $\%$ or median & P25, P75 & \\
\hline Male & 439 & $51 \cdot 5$ & & 332 & 51.5 & & 115 & $56 \cdot 5$ & & 0.602 \\
\hline Low social class & 437 & 35.9 & & 330 & $38 \cdot 8$ & & 114 & $45 \cdot 6$ & & 0.161 \\
\hline Family history of diabetes & 438 & 3.65 & & 328 & 3.66 & & 114 & 7.02 & & 0.239 \\
\hline BMI $\left(\mathrm{kg} / \mathrm{m}^{2}\right)$ & 439 & $19 \cdot 4$ & $17 \cdot 9,20 \cdot 9$ & 332 & 19.5 & $18 \cdot 2,21 \cdot 4$ & 115 & $20 \cdot 0$ & $18 \cdot 5,21 \cdot 7$ & 0.018 \\
\hline Daily intake of sweets & 438 & $10 \cdot 0$ & & 331 & $21 \cdot 8$ & & 115 & $24 \cdot 3$ & & $<0.001$ \\
\hline High alcohol intake & 437 & $10 \cdot 8$ & & 328 & $23 \cdot 8$ & & 115 & 43.5 & & $<0.001$ \\
\hline Smoking (\%) & 438 & $13 \cdot 7$ & & 331 & 31.4 & & 115 & $55 \cdot 6$ & & $<0.001$ \\
\hline Physical activity > once/week & 439 & $58 \cdot 1$ & & 332 & 41.6 & & 115 & 30.4 & & $<0.001$ \\
\hline
\end{tabular}

Data are presented as $n$ and \% except for BMI, where data are presented as median and P25 (25th percentile), P75 (75th percentile).

Table 2 Spearman's rank correlations $\left(R_{\mathrm{s}}\right)$ between regularity in separate meal habits and covariates at age 16 years in 889 participants from the Northern Swedish Cohort

\begin{tabular}{|c|c|c|c|}
\hline & Eating breakfast & Regularly school lunch & Regularly dinner with family \\
\hline Regularly school lunch & $0.18^{\star \star \star}$ & - & - \\
\hline Regularly dinner with family & $0.09^{\star *}$ & $0.09^{* *}$ & - \\
\hline Male sex & -0.06 & 0.003 & 0.001 \\
\hline Low social class & -0.01 & -0.05 & -0.05 \\
\hline Family history diabetes & $-0.08^{*}$ & -0.02 & -0.03 \\
\hline BMI & -0.06 & -0.06 & $-0.08^{*}$ \\
\hline Daily sweets & -0.03 & $-0 \cdot 18^{\star \star *}$ & -0.06 \\
\hline High alcohol intake & $-0 \cdot 14^{\star \star *}$ & $-0.23^{\star \star \star}$ & $-0 \cdot 14^{\star \star *}$ \\
\hline Smoking & $-0.20^{\star * *}$ & $-0.25^{\star \star \star}$ & $-0 \cdot 15^{\star \star \star}$ \\
\hline Physical activity >once/week & $0.16^{\star \star *}$ & $0 \cdot 18^{\star \star \star}$ & 0.05 \\
\hline
\end{tabular}

${ }^{\star} P<0.05,{ }^{* \star} P<0.01,{ }^{* \star \star} P<0.001$.

Table 3 Logistic regression results for associations between meal regularity at age 16 years and the metabolic syndrome at age 43 years for participants from the Northern Swedish Cohort

\begin{tabular}{|c|c|c|c|c|c|c|c|c|c|}
\hline & \multirow[b]{2}{*}{$n$} & \multicolumn{2}{|c|}{ Unadjusted } & \multicolumn{2}{|c|}{ Model $1^{*}$} & \multicolumn{2}{|c|}{ Model $2 \dagger$} & \multicolumn{2}{|c|}{ Model 3‡ } \\
\hline & & OR & $95 \% \mathrm{Cl}$ & OR & $95 \% \mathrm{Cl}$ & OR & $95 \% \mathrm{Cl}$ & OR & $95 \% \mathrm{Cl}$ \\
\hline All meals regularly & 439 & 1.00 & Ref. & 1.00 & Ref. & 1.00 & Ref. & 1.00 & Ref. \\
\hline One meal irregularly & 332 & $1 \cdot 19$ & $0.85,1.65$ & $1 \cdot 19$ & $0.85,1.66$ & $1 \cdot 13$ & $0.80,1.59$ & 0.97 & $0.68,1.39$ \\
\hline Two or all meals irregularly & 115 & 1.74 & $1 \cdot 12,2 \cdot 71$ & $1 \cdot 70$ & $1.09,2.66$ & 1.54 & $0.97,2.44$ & 1.18 & $0.71,1.95$ \\
\hline$P$ for trend§ & & 0.008 & & 0.010 & & 0.048 & & 0.476 & \\
\hline
\end{tabular}

Ref., reference category.

${ }^{*}$ Model $1=$ adjusted for sex.

†Model $2=$ model 1 plus adjustment for BMl at age 16 years

¥Model $3=$ model 2 plus adjustment for smoking, alcohol consumption, physical activity and consumption of sweets and pastries at age 16 years.

$\S P$ for trend for none to three irregular meals. 
significant after adjustment for BMI at age 16 years (model 2) and further attenuated after addition of lifestyle habits at age 16 years (model 3; Table 3). Further adjustment for social class and family history of diabetes did not affect the results (data not shown; OR $=1.17$; $95 \%$ CI $0.70,1.95)$.

To investigate which of the lifestyle-related variables contributed to the attenuation of the relationship between meal regularity at age 16 years and the metabolic syndrome at age 43 years, additional analyses were performed in which the variables were added to the sex-adjusted model (model 1, in Table 3), in separate analyses for each covariate. All of the covariates BMI, smoking status, high alcohol consumption and physical activity individually attenuated the association at a similar degree, and addition of each resulted in a non-significant association between the exposure and the outcome. Addition of daily consumption of sweets and pastries did not attenuate the association (data not shown).

Analyses on the relationship between regularity in the specific meal habits at age 16 years and the metabolic syndrome at age 43 years are shown in Table 4. Poor breakfast was the only separate meal habit in adolescence associated with significantly higher prevalence of the metabolic syndrome in adulthood, although the results for both lunch and dinner pointed in the same direction but more weakly. This effect of poor breakfast also remained after adjustment for the other two meal habits, sex, BMI and lifestyle habits at age 16 years.

Replacing dichotomized variables with continuous ones (where possible) did not evidently affect the relationship between overall meal regularity or specific main meal regularity at age 16 years and the metabolic syndrome at age 43 years.

The next series of analyses focused on the relationship between meal regularity in adolescence and the separate components of the metabolic syndrome (i.e. central obesity, high TAG, low HDL-cholesterol, high blood pressure and high fasting glucose) in adulthood. As for the components of the metabolic syndrome, irregular eating of meals at age 16 years was associated with central obesity and high fasting glucose at age 43 years in sex-adjusted analysis, but these associations were not statistically significant after adjustments for BMI at age 16 years or lifestyle at age 16 years (central obesity, model 3: $\mathrm{OR}=1.23 ; 95 \% \mathrm{CI} 0.76,2.00 ;$ high fasting glucose, model 3: OR $=1 \cdot 30 ; 95 \%$ CI $0.74,2 \cdot 26$; see online supplementary material, Supplemental Table 1). In multivariable analysis for the specific meals, poor breakfast habits at age 16 years was borderline significantly associated with central obesity at age 43 years (model 4: OR $=1.71 ; 95 \%$ CI 0.99, 2.94; Supplemental Table 2).

\section{Discussion}

The present study found that irregular eating of meals at age 16 years was associated with higher prevalence of the metabolic syndrome at age 43 years, but the association was largely explained by early BMI and lifestyle habits. BMI, smoking, high alcohol intake and low level of physical activity at age 16 years contributed to the attenuation of the association at a similar level. Of the meals, poor breakfast was the only separate meal associated with future metabolic syndrome, also after adjustment for the other two meals, BMI and early lifestyle factors. Poor breakfast at age 16 years was close to statistically significantly associated with central obesity at age 43 years in the fully adjusted model.

The finding of an association between poor breakfast consumption in adolescence and higher risk of the metabolic syndrome in adulthood in this population has been previously published and discussed ${ }^{(10)}$. In the current study we show that the association between poor breakfast in adolescence and the metabolic syndrome in adulthood remains also after adjustment for the other meal habits (school lunch and dinner with family). This indicates that breakfast is the meal in adolescence with the largest importance for future metabolic health. A special protective role of breakfast eating on cardiometabolic risk is supported by previous experimental and observational studies $^{(8,21-25)}$. It cannot be ruled out that the other meals examined here (school lunch and dinner with family) have other important health consequences in the short run. There is a rather strong correlation between skipping school lunch and higher consumption of sweets and

Table 4 Logistic regression results for associations between eating specific meals irregularly at age 16 years and the metabolic syndrome at age 43 years for participants from the Northern Swedish Cohort

\begin{tabular}{|c|c|c|c|c|c|c|c|c|c|c|c|c|}
\hline & \multirow[b]{2}{*}{$n / N$} & \multirow[b]{2}{*}{$\%$} & \multicolumn{2}{|c|}{ Unadjusted } & \multicolumn{2}{|c|}{ Model $1^{*}$} & \multicolumn{2}{|c|}{ Model $2 \dagger$} & \multicolumn{2}{|c|}{ Model 3† } & \multicolumn{2}{|c|}{ Model $4 \S$} \\
\hline & & & OR & $95 \% \mathrm{Cl}$ & OR & $95 \% \mathrm{Cl}$ & OR & $95 \% \mathrm{Cl}$ & OR & $95 \% \mathrm{Cl}$ & OR & $95 \% \mathrm{Cl}$ \\
\hline Poor t & $88 / 889$ & 9.9 & $2 \cdot 25$ & $1.43,3.54$ & $2 \cdot 12$ & $1.33,3.38$ & $2 \cdot 02$ & $1 \cdot 25,3.23$ & 1.96 & $1 \cdot 20,3 \cdot 20$ & 1.67 & $1 \cdot 00,2 \cdot 80$ \\
\hline School lunch irregularly & $365 / 888$ & 41 & 1.16 & $0.86,1.57$ & 1.05 & $0.76,1.43$ & 1.06 & $0.77,1.45$ & 1.00 & $0.72,1.39$ & 0.87 & $0.61,1.22$ \\
\hline Dinner with family irregularly & $127 / 887$ & 14 & 1.30 & $0.86,1.97$ & 1.23 & $0.81,1.87$ & 1.25 & $0.81,1.90$ & $1 \cdot 15$ & $0.74,1.78$ & 1.11 & $0.71,1.74$ \\
\hline
\end{tabular}

*Model $1=$ adjusted for the other two meal habits at age 16 years.

†Model $2=$ model 1 plus adjustment for sex.

$\ddagger$ Model $3=$ model 2 plus adjustment for BMl at age 16 years.

$\S$ Model $4=$ model 3 plus adjustment for smoking, alcohol consumption, physical activity and consumption of sweets and pastries at age 16 years. 
pastries, which may be a consequence of the skipped lunch meal. An association between healthier school food at lunch and higher alertness has been found ${ }^{(26)}$, and this may be of importance for future academic achievement and thereby future socio-economic circumstances.

Family meals have been associated with risk prevention (less smoking, alcohol and drugs) in several previous studies, although the direction of the association requires further research ${ }^{(27)}$. In our study there were significant correlations between frequency of dinner with family and less smoking as well as lower prevalence of high alcohol consumption. However, these risk behaviours evidently correlated higher with both breakfast and frequency of school lunch, where also a clear correlation was found with physical activity.

The prospective design and the high participation rate in follow-up are the major strengths within the present study. The study also has some limitations. We do not have complete information on meal frequency, which has been the exposure variable in many previous studies; we have information on main meals, but no information on in-between meals. As for breakfast, we have data on breakfast on the day of examination only. This may decrease the measurement precision, but would rather lead to underestimation than overestimation of the association $^{(28)}$. We do not know if those skipping school lunch eat lunch somewhere else (at home, at a restaurant, etc.), but the correlation between skipping school lunch and daily consumption of sweets and pastries indicates that school lunch is often replaced with less healthy options. Few countries offer a free, cooked meal for lunch in school, making the findings concerning school lunch difficult to compare with other countries. We do not know if those not eating dinner with family regularly have dinner by themselves or together with someone else. The limitations concerning estimation of lunch and dinner may have attenuated any association between irregularity in eating meals at age 16 years and the metabolic syndrome at age 43 years. Furthermore, key variables were self-reported and there may be some degree of misclassification.

The inference remains that we were unable to attribute any significant contribution of meal regularity independently of other lifestyle factors. As a corollary, we cannot find support for regular eating of meals per se being important for long-term cardiometabolic health. Considering that poor breakfast was associated with the risk of future metabolic syndrome also after multivariate adjustments, breakfast eating in adolescence should be promoted.

\section{Conclusions}

Irregular eating of meals in adolescence predicted the metabolic syndrome in adulthood, but the association was not independent of BMI and lifestyle in adolescence. Poor breakfast in adolescence, but not irregular eating of school lunch or dinner with family, was associated with future metabolic syndrome, independent of BMI and lifestyle in adolescence. These results indicate that although irregular eating of all meals may not involve long-term cardiometabolic health risks, special effort should be put on encouraging breakfast eating in adolescence.

\section{Acknowledgements}

Financial support: The work was supported through a regional agreement between Umeå University and Västerbotten County Council on cooperation in the field of Medicine, Odontology and Health (ALF). ALF had no role in the design, analysis or writing of this article. Conflict of interest: None. Authorship: M.W. formulated the research question; A.H. designed the research; A.H. conducted the research (data collection); M.W. analysed the data; M.W., P.E.G., P.W. and A.H. interpreted the data; M.W. wrote the paper; P.E.G, P.W. and A.H. critically revised the paper; all authors read and approved the final manuscript. Ethics of buman subject participation: This study was conducted according to the guidelines laid down in the Declaration of Helsinki and all procedures involving human subjects were approved by the Regional Ethical Review Board in Umeå. Separate written informed consent was not requested by the Ethical Review Board, as the participants were regarded as giving written consent when completing the questionnaire at each data collection wave. All participants were clearly informed that participation in the study is voluntary and that they can decide to withdraw from participation at any time, without giving any explanation.

\section{Supplementary material}

To view supplementary material for this article, please visit http://dx.doi.org/10.1017/S1368980015001445

\section{References}

1. Nordic Council of Ministers (2005) Nordic Nutrition Recommendations, NNR 2004: Integrating Nutrition and Physical Activity, pp. 85-101. Copenhagen: Nordic Council of Ministers.

2. Kruger J, Galuska DA, Serdula MK et al. (2004) Attempting to lose weight: specific practices among US adults. Am J Prev Med 26, 402-406.

3. Sierra-Johnson J, Unden AL, Linestrand $\mathrm{M}$ et al. (2008) Eating meals irregularly: a novel environmental risk factor for the metabolic syndrome. Obesity (Silver Spring) 16, 1302-1307.

4. Smith KJ, Blizzard L, McNaughton SA et al. (2012) Daily eating frequency and cardiometabolic risk factors in young Australian adults: cross-sectional analyses. Br J Nutr 108, 1086-1094. 
5. Titan SM, Bingham S, Welch A et al. (2001) Frequency of eating and concentrations of serum cholesterol in the Norfolk population of the European prospective investigation into cancer (EPIC-Norfolk): cross sectional study. BMJ 323, 1286-1288.

6. Speechly DP \& Buffenstein R (1999) Greater appetite control associated with an increased frequency of eating in lean males. Appetite 33, 285-297.

7. Farshchi HR, Taylor MA \& Macdonald IA (2005) Beneficial metabolic effects of regular meal frequency on dietary thermogenesis, insulin sensitivity, and fasting lipid profiles in healthy obese women. Am J Clin Nutr 81, 16-24.

8. Pot GK, Hardy R \& Stephen AM (2014) Irregular consumption of energy intake in meals is associated with a higher cardiometabolic risk in adults of a British birth cohort. Int J Obes (Lond) 38, 1518-1524.

9. Wennberg P, Gustafsson PE, Dunstan DW et al. (2013) Television viewing and low leisure-time physical activity in adolescence independently predict the metabolic syndrome in mid-adulthood. Diabetes Care 36, 2090-2097.

10. Wennberg M, Gustafsson PE, Wennberg P et al. (2014) Poor breakfast habits in adolescence predict the metabolic syndrome in adulthood. Public Health Nutr (Epublication ahead of print version).

11. Hammarstrom A \& Janlert U (2011) Cohort profile: the northern Swedish cohort. Int J Epidemiol 41, 1545-1552.

12. Gustafsson PE, Persson M \& Hammarstrom A (2011) Life course origins of the metabolic syndrome in middle-aged women and men: the role of socioeconomic status and metabolic risk factors in adolescence and early adulthood. Ann Epidemiol 21, 103-110.

13. Gustafsson PE, Persson M \& Hammarstrom A (2012) Socioeconomic disadvantage and body mass over the life course in women and men: results from the Northern Swedish Cohort. Eur J Public Health 22, 322-327.

14. Alberti KG, Zimmet P \& Shaw J (2005) The metabolic syndrome - a new worldwide definition. Lancet $\mathbf{3 6 6}$, $1059-1062$.

15. Hammarstrom A (1986) Youth unemployment and ill-health: results from a two year follow-up study. Doctoral Thesis, Karolinska Institute (in Swedish, with an English summary).

16. Janlert U \& Hammarstrom A (1992) Alcohol consumption among unemployed youths: results from a prospective study. BrJ Addict 87, 703-714.
17. Hammarstrom A \& Janlert U (1994) Unemployment and change of tobacco habits: a study of young people from 16 to 21 years of age. Addiction 89, $1691-1696$.

18. Gustafsson PE \& Hammarstrom A (2012) Socioeconomic disadvantage in adolescent women and metabolic syndrome in mid-adulthood: an examination of pathways of embodiment in the Northern Swedish Cohort. Soc Sci Med 74, 1630-1638.

19. Stocks T, Bjorge $\mathrm{T}$, Ulmer $\mathrm{H}$ et al. (2015) Metabolic risk score and cancer risk: pooled analysis of seven cohorts. Int J Epidemiol (Epublication ahead of print version).

20. Eckel RH, Grundy SM \& Zimmet PZ (2005) The metabolic syndrome. Lancet 365, 1415-1428.

21. Farshchi HR, Taylor MA \& Macdonald IA (2005) Deleterious effects of omitting breakfast on insulin sensitivity and fasting lipid profiles in healthy lean women. Am J Clin Nutr 81, 388-396.

22. Smith KJ, Gall SL, McNaughton SA et al. (2010) Skipping breakfast: longitudinal associations with cardiometabolic risk factors in the Childhood Determinants of Adult Health Study. Am J Clin Nutr 92, 1316-1325.

23. Mekary RA, Giovannucci E, Willett WC et al. (2012) Eating patterns and type 2 diabetes risk in men: breakfast omission, eating frequency, and snacking. Am J Clin Nutr 95, 1182-1189.

24. Mekary RA, Giovannucci E, Cahill L et al. (2013) Eating patterns and type 2 diabetes risk in older women: breakfast consumption and eating frequency. Am J Clin Nutr 98, 436-443.

25. Cahill LE, Chiuve SE, Mekary RA et al. (2013) Prospective study of breakfast eating and incident coronary heart disease in a cohort of male US health professionals. Circulation 128, 337-343.

26. Golley R, Baines E, Bassett P et al. (2010) School lunch and learning behaviour in primary schools: an intervention study. Eur J Clin Nutr 64, 1280-1288.

27. Goldfarb S, Tarver WL \& Sen B (2014) Family structure and risk behaviors: the role of the family meal in assessing likelihood of adolescent risk behaviors. Psychol Res Behav Manag 7, 53-66.

28. Carroll R (1998) Measurement error in epidemiologic studies. In Encyclopedia of Biostatistics, pp. 2491-2519 [P Armitage and T Colton, editors]. Chichester: Wiley. 